\title{
Effects of high solenoidal magnetic fields on rf accelerating cavities
}

\author{
A. Moretti and Z. Qian \\ Fermi National Accelerator Laboratory, Batavia, Illinois 60510, USA \\ J. Norem* \\ Argonne National Laboratory, Argonne Illinois 60439, USA \\ Y. Torun \\ Illinois Institute of Technology, Chicago, Illinois 60616, USA \\ D. Li and M. Zisman \\ Lawrence Berkeley National Laboratory, Berkeley, California 94720, USA
}

(Received 18 April 2005; published 6 July 2005; corrected 7 September 2005)

\begin{abstract}
We have measured the effects of high $(0-4.5 \mathrm{~T})$ magnetic fields on the operating conditions of $805 \mathrm{MHz}$ accelerating cavities, and discovered that the maximum accelerating gradient drops as a function of the axial magnetic field. While the maximum gradient of any cavity is governed by a number of factors including conditioning, surface topology and materials, we argue that $\mathbf{J} \times \mathbf{B}$ forces within the emitters are the mechanism for enhanced breakdown in magnetic fields. The pattern of emitters changes over time and we show an example of a bright emitter which disappears during a breakdown event. We also present unique measurements of the distribution of enhancement factors, $\beta$, of secondary emitters produced in breakdown events during conditioning. We believe these secondary emitters can also be breakdown triggers, and the secondary emitter spectrum helps to determine the maximum operating field.
\end{abstract}

DOI: $10.1103 /$ PhysRevSTAB.8.072001

PACS numbers: 29.17.+w, 52.80.Vp, 73.22.-f

\section{INTRODUCTION}

Cooling beams of muons in flight requires absorbers to reduce the muon momentum, accelerating fields to replace the lost momentum, and static solenoidal magnetic fields to focus the muon beams $[1,2]$. The process is most efficient if both the magnetic fields and accelerating fields are high. In order to study the interactions of a static magnetic field with the operation of high gradient accelerating fields we have conducted tests to determine the operating envelope of accelerating cavities in high magnetic fields. These studies have already produced useful information on dark currents, the environment of field emitters in cavities, conditioning and breakdown mechanisms [3]. This paper discusses measurements made to determine the highest operating field compatible with a given magnetic field and understand the mechanisms limiting this operation.

\section{MEASUREMENTS}

Two $805 \mathrm{MHz}$ cavities were used, one was a six-cell standing wave structure $1 \mathrm{~m}$ long, and the other was a simple pillbox, $8.64 \mathrm{~cm}$ long, which had replaceable end windows to terminate the electric field. These cavities were operated on the axis of a solenoidal field created by superconducting Helmholtz coils mounted in a structure with a warm bore of $0.44 \mathrm{~m}$. The geometry was described in Ref. [3]. Both cavities were operated at maximum surface fields from 40 to $60 \mathrm{MV} / \mathrm{m}$.

*Electronic address: norem@anl.gov
While the majority of the operation was done either with no field or $B<2.5 \mathrm{~T}$, the coil system was operated at up to $4.5 \mathrm{~T}$ with the fields adding, producing a solenoidal field, and with the coils bucking each other, which produced a gradient of up to $20 \mathrm{~T} / \mathrm{m}$ at the center of the magnet. Because of the size of the six-cell cavity, only one end of this cavity was exposed to the maximum field. The majority of the results from operation of the six-cell cavity in the field, describing changes in the radiation environment, field emitted dark current orbits and cavity damage have been published [3]. The geometry of the six-cell cavity was complex, with oblate spheroidal cells separated by blunt irises where the magnetic field and electric fields were, in general, not parallel.

The pillbox cavity, shown in Fig. 1, was different in a number of ways. Because of the flat walls, the electric and magnetic fields were roughly parallel throughout the cavity. Removable windows permitted study of the breakdown damage with a variety of materials. Thin $(0.254 \mathrm{~mm}) \mathrm{Be}$ windows, used for most of the operation, permitted study of field emitted electron beams, which in turn produced information on the structure of the surface. We could watch the spatial distribution of secondary field emission sources change over a period of weeks. The conditioning process to reach full field (arbitrarily limited at $40 \mathrm{MV} / \mathrm{m}$ ) took on the order of a week after passing through low power multipacting zones. Some results from measurements using this cavity were presented at PAC03 $[4,5]$.

Data taken over a period of 6 months with different magnetic configurations are shown in Fig. 2 [6]. We plot 


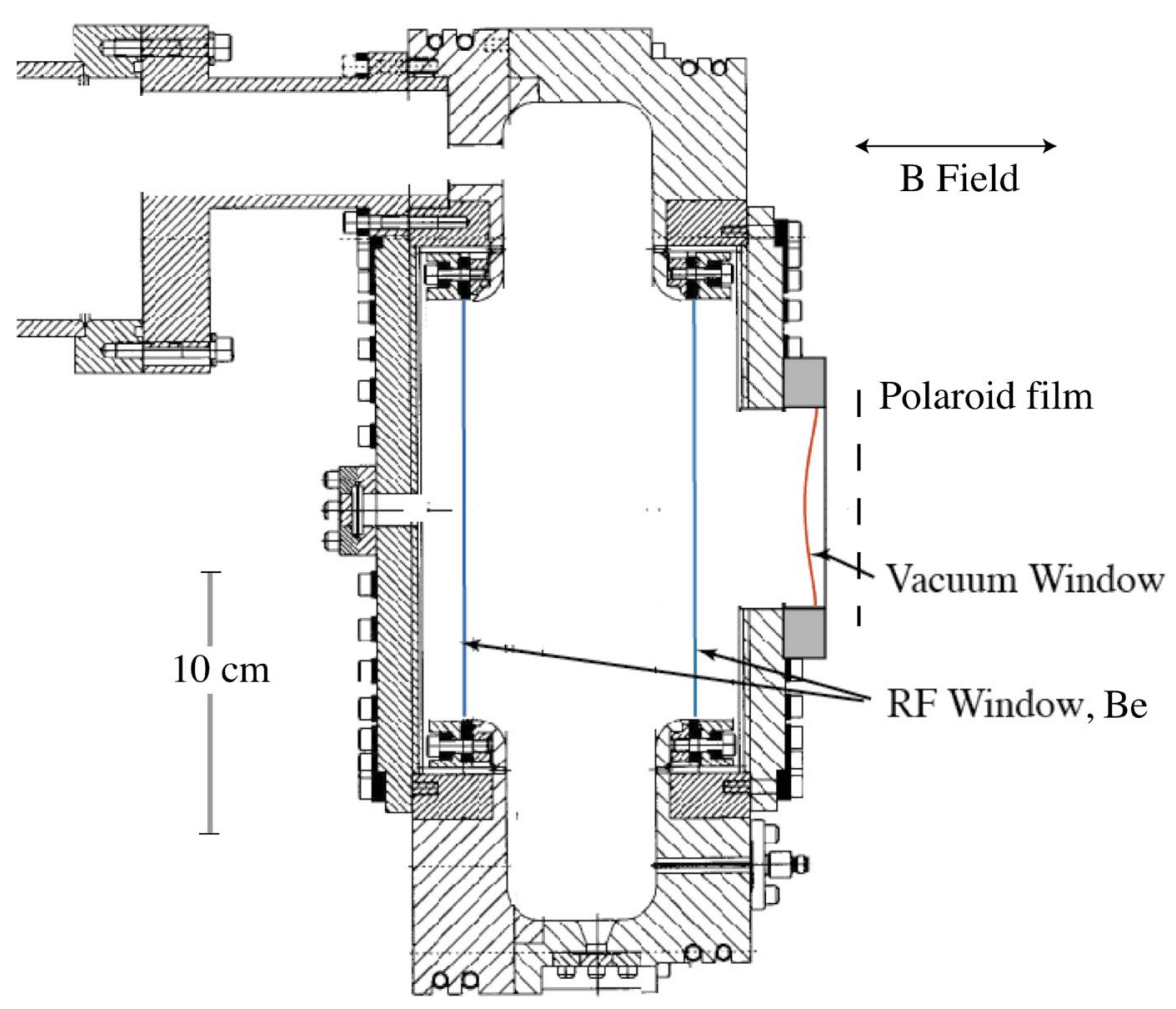

FIG. 1. (Color) The pillbox cavity showing $0.254 \mathrm{~mm}$ Be rf windows and $0.2 \mathrm{~mm}$ Ti vacuum window.

here the electric field achieved as a function of the static solenoidal field for two coils in parallel, two coils bucking and one coil. Since we have already shown that solenoidal fields affect conditioning time, an attempt was made to condition as long as possible at the highest fields, however this was difficult due to the cost of operating the superconducting magnet. Conditioning with magnetic fields seemed to be somewhat less stable than without the fields, as breakdown events required a somewhat longer recovery time. Thus the cavity may not be adequately conditioned at the highest magnetic fields. The general trend of the data, consistent with all measurements made, is that the accelerating field is degraded by the presence of the magnetic field. The maximum accelerating field is limited by the conditioning process and by the mechanism of breakdown in magnetic fields, which we will describe below.

The data shown in Fig. 2 were taken before we understood what the limitations imposed by the magnetic field might be, and may not have used an optimum algorithm. The time required for conditioning the cavity with no magnetic field can be seen in Fig. 3, which shows the radiation level detected in a monitor as a function of time, for operation at constant accelerating field. Since the time required to significantly improve the cavity performance is a few days, and the total operating time of the superconducting solenoid was limited by the cost of helium, it seems desirable to operate much longer at fewer magnetic field settings, and this will be done in the future experiments.

\section{A. Breakdown with electric and magnetic fields}

With no magnetic field, we have shown that breakdown can occur when local tensile stresses exerted by electric fields are greater than the tensile strength of the material

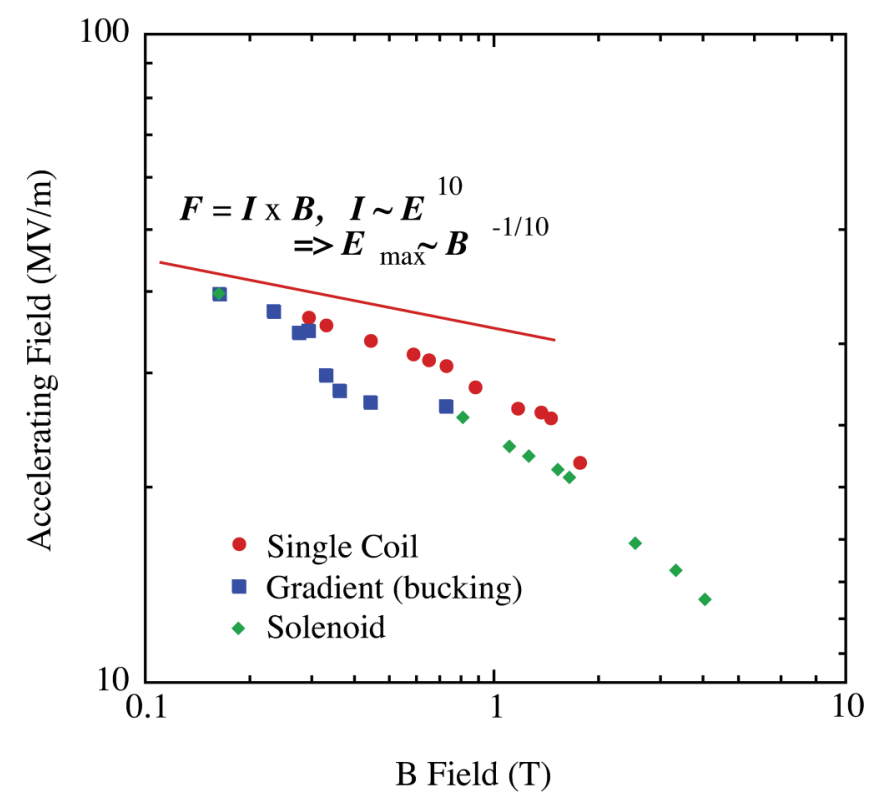

FIG. 2. (Color) Breakdown threshold as a function of static magnetic field at the cavity surfaces for different magnetic configurations. 


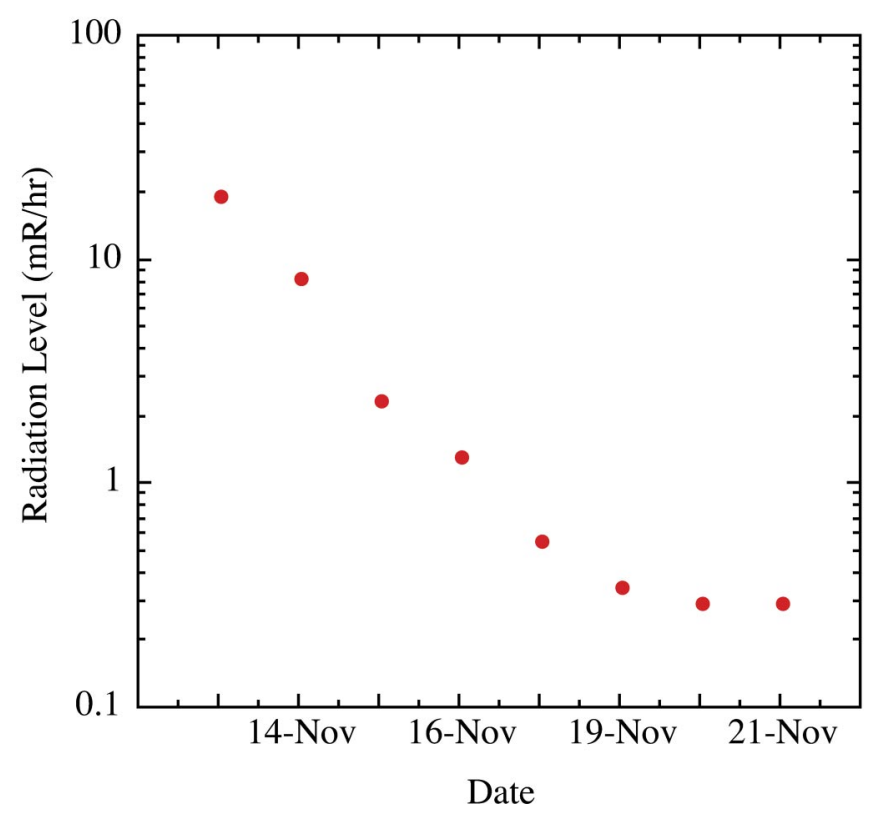

FIG. 3. (Color) Radiation level near the cavity as a function of time for $B=0$.

[3,7]. We believe magnetic fields can facilitate breakdown by exerting additional forces in the form of torques on field emitters. Assuming that emitters are basically conical, and aligned roughly parallel to a magnetic field, the current density at the surface of an emitter is $j=J / A$, where $J$ is the maximum measured current per emitter, on the order of $0.1 \mathrm{~mA}$ for the six-cell cavity, and $A$, the local emitter area of the order $10^{-14} \mathrm{~m}^{2}$, giving current densities of $10^{10} \mathrm{~A} / \mathrm{m}^{2}[3,7]$. The radial component of this current density, perpendicular to the magnetic field, is determined by the cone angle of the emitter, $\theta$, so the perpendicular pressure exerted by the magnetic field is then $\sin (\theta) J B / A$, since the stress is due to $\mathbf{j} \times \mathbf{B}$ forces, as in Fig. 4. With a $2 \mathrm{~T}$ magnetic field and $0.1 \mathrm{~mA}$ emitter this pressure can be on the order of $10000 \mathrm{MPa}$, more than enough to trigger fracture. The forces will be directed in a circular pattern around the emitter tip, with a smooth gradient in sheer.

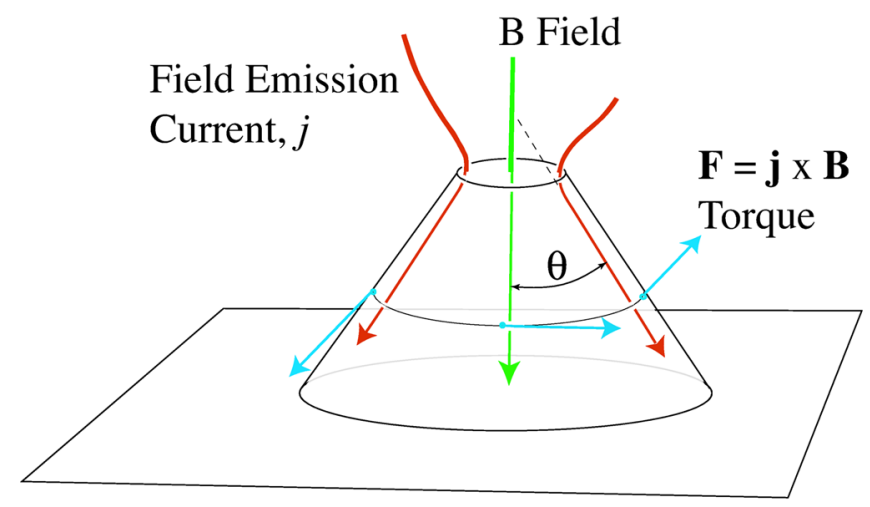

FIG. 4. (Color) Forces due to field emission currents are present in the field emitters.
If breakdown occurs as a result of a limiting pressure on a given emitter, one can derive a relation between the maximum electric field and the static magnetic field from the expression $j B=$ const. As we have shown in Ref. [3], the relation between current and field is approximately $j \propto$ $E^{10}$ in the range where breakdown occurs. This implies a dependence of $E_{\max } \propto B^{-1 / 10}$ for emitters near this limit, where $E_{\max }$ is the breakdown limited maximum field. Data from the pillbox cavity seem to be constrained by this relation at low field, but depart somewhat from the prediction at high fields, where less time was spent conditioning and the maximum field was not reached.

If this model is correct, it may be straightforward to prevent breakdown caused by this process. Since the mechanism that couples the magnetic field to breakdown of the cavity seems to be $\mathbf{J} \times \mathbf{B}$ forces driven by field emission of electrons, thin coatings of high work function materials would reduce the field emission and the local pressure on the emitter tips. Control of field emission with thin layers of a material with different work function was demonstrated with tungsten emitters over 50 years ago [8]. While it is, in principle, possible to reduce field emission by making the interior of cavities arbitrarily smooth, the conditioning process can roughen the surface, and we would prefer to develop a method that can cope with problems in operating cavities.

In the open cell cavity, the electric field was not, in general, parallel to the magnetic field as shown in Fig. 4. We noticed that at high magnetic fields $(\sim 3 \mathrm{~T})$ this cavity required extensive reconditioning whenever the static magnetic field was turned on or off. With the pillbox cavity, where the electric and magnetic fields were roughly parallel, less conditioning was required to reach higher electric fields in the presence of solenoidal fields. We assume the difference in the behavior of the two cavities could be due to the local orientation of field emitters relative to the magnetic field. This will be examined in a variety of future experiments.

\section{B. Field emitters and breakdown triggers}

In a magnetic field, dark currents will follow field lines. With strong solenoidal magnetic fields it is possible to identify individual emitters in the cavity and to monitor changes in the pattern of field emitters with time, using Polaroid [9] $4 \times 5$ sheet film and larger sheets of photographic paper [10] to record the dark currents at the surface of the vacuum window. The dark currents are scattered by both the Be rf windows and the vacuum windows increasing the beam diameter. Since the cavity was first conditioned without magnetic field, the pattern of emitters was well developed when the first Polaroid pictures were made with low field. We could observe changes in this pattern over times on the order of weeks, when emitters would appear or disappear. 


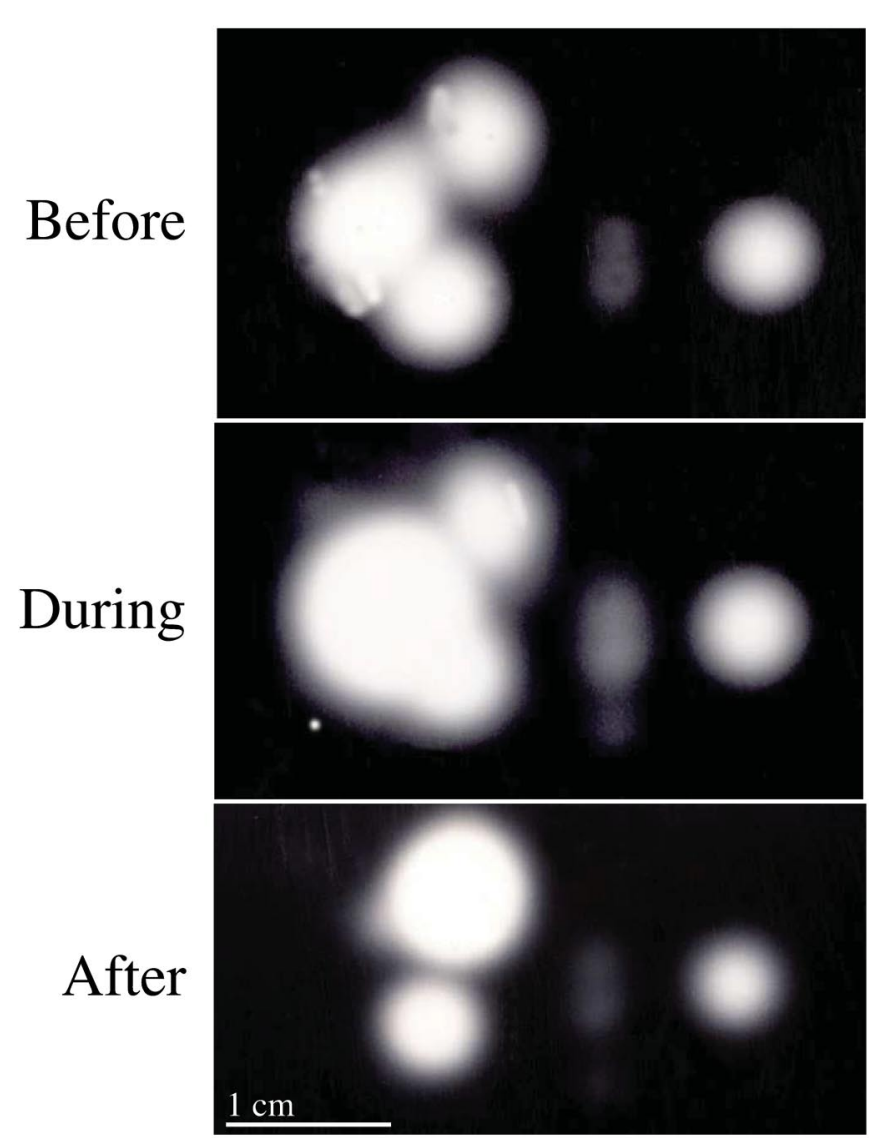

FIG. 5. Polaroid pictures, taken over a $10 \mathrm{~min}$ period, of an emitter pattern before, during and after a breakdown event.

We were able to capture one breakdown event on film. The pictures, shown in Fig. 5, were made at low B field, i.e., low resolution. The first picture shows the initial distribution, before the spark, with one of the emitters brighter than the rest. The middle picture shows the brightest emitter becoming more active, which we assume is associated with a breakdown event that took place during this exposure. The final picture shows the pattern of emitters after the breakdown event. At this time the brightest emitter is gone, we assume it was the trigger of the breakdown event. (The first exposure was slightly damaged during development.)

The Polaroid exposures were about 1 min long and the series was taken in about $10 \mathrm{~min}$, which determines the time resolution of this technique. We believe that this series of pictures demonstrates how active emitters, experiencing high fields, can be the source of breakdown triggers. We are developing methods of viewing this pattern with better spatial and time resolution.

\section{The spectrum of field emitters}

We have previously described a model of cavity conditioning [3]. When a new cavity is produced, we assume the surface has a distribution of asperities with a wide range of enhancement factors. As the power level is slowly raised, the hotter emitters are burned off and the average field that can be maintained in the cavity is increased. As each emitter is burned away, however, it produces a distribution of secondary emitters on the surface which may also require conditioning. Ultimately the maximum operating field that can be generated by a cavity is the equilibrium field where the production of secondary emitters maintains itself.

Operation in the magnetic field allowed us to measure the distribution of secondary emission sources produced in breakdown events. These measurements were obtained by comparing the density of field emitted electron beams produced from the Be windows in Polaroid photographs taken at different accelerating fields. The relative emitter strength was measured over many orders of magnitude in emitted current by digitizing the photographs, as in Fig. 6 . After the Be windows were removed from the cavity, they were examined with an electron microscope and the beryllium surface, which was undamaged, was found to be covered with copper splashes, which we believe to be the result of breakdown events from the nearby copper.

These splashes are presumed to be the source of the field emitted beams. The spectrum of emitters produced from the Be windows, shown in Fig. 7, is then the spectrum of secondary sources. The points with high enhancement factors were identified from the photographs of beamlets, with the maximum enhancement factor normalized by the assumption that the local field would be about $8 \mathrm{GV} / \mathrm{m}$, where we have found breakdown events (like those in Fig. 5) can occur [3]. The point at low enhancement factors was identified by measuring the densities, shapes, and dimensions of deposited copper splashes obtained from electron microscope images. Assuming that the splashes are roughly spherical or half spherical, enhancement factors of 3-4 can be calculated from the geometry alone [11]. It is difficult to determine with any precision if the splashes have areas with higher enhancement factors from microscope images. The line on the plot does not imply the distribution is known in the region between the points. These data are consistent with a variety of models; the line shows a distribution that is proportional to $e^{-0.027 \beta}$, and we would expect this spectrum to be strongly dependent on the cavity stored energy.

This distribution shows that there are many more weak emitters per unit enhancement than large ones, consistent with visual and SEM inspection of the Be windows, which showed many copper splashes and fragments, only a small fraction of which seemed to be field emission sources.

\section{SUMMARY}

Measurements of the maximum accelerating field possible in the presence of a static magnetic field show that the magnetic field can limit the operating range of the cavity. We argue that the mechanism responsible is $\mathbf{j} \times \mathbf{B}$ forces 

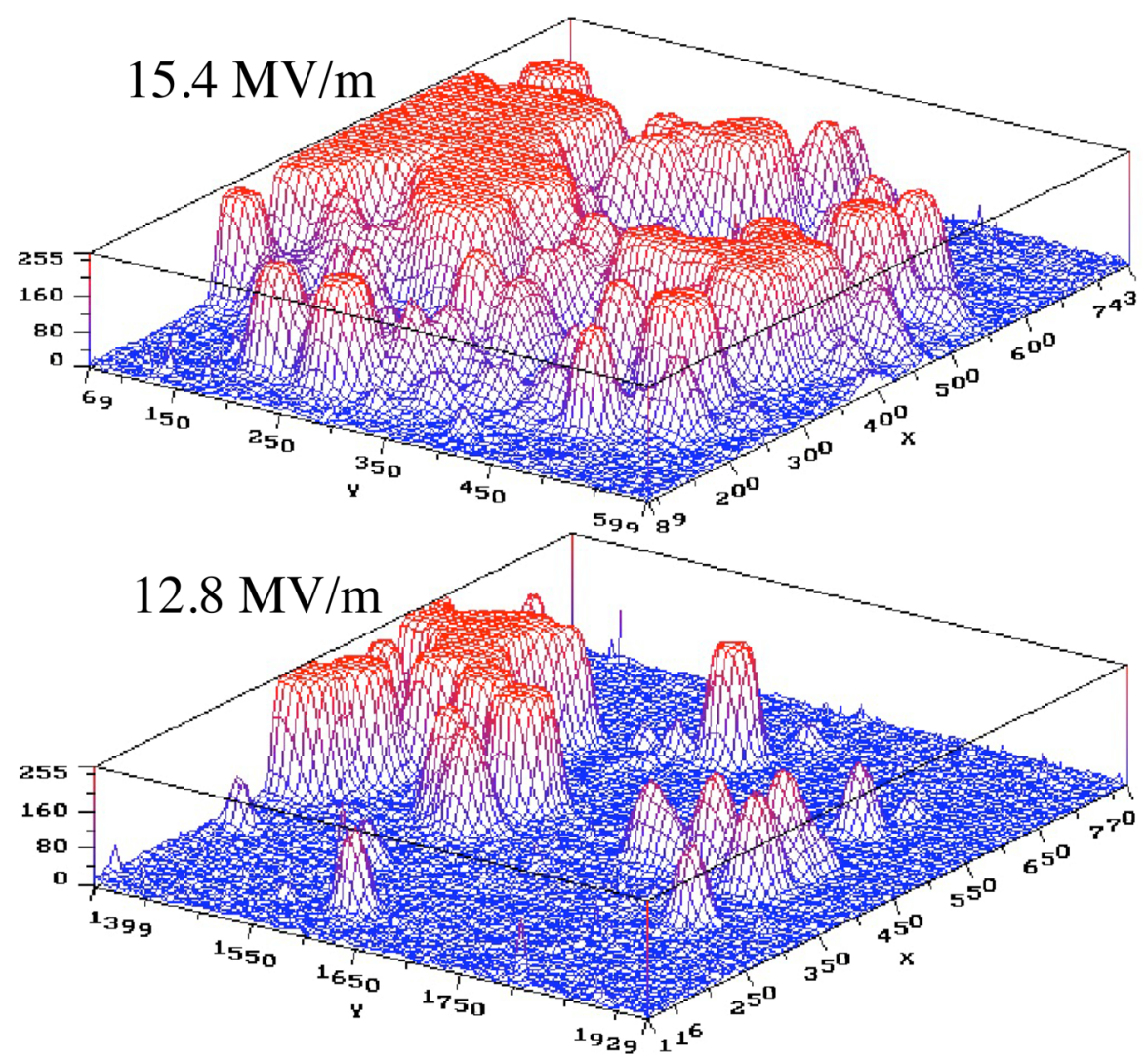

FIG. 6. (Color) Digitized images of the field emitted currents at different gradients. The digitized area is $7 \mathrm{by} 7 \mathrm{~cm}$.

within the emitters. This simple model can explain the general behavior of the decline in electric field with magnetic field. We have also used the magnetic field to show how the conditioning process produces secondary field

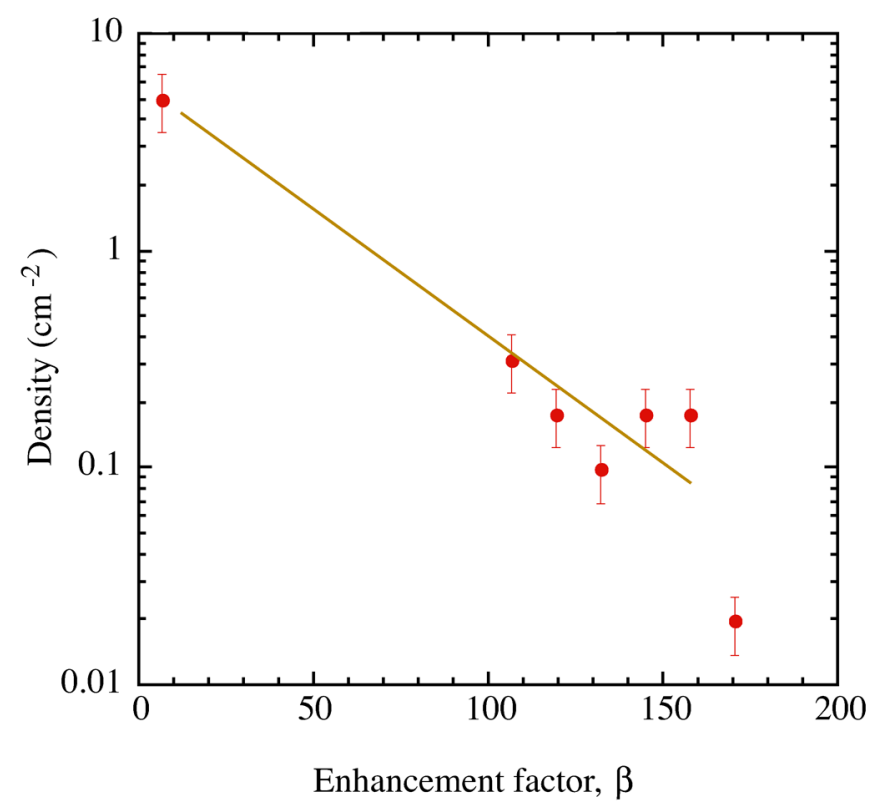

FIG. 7. (Color) The spectrum of secondary emitters produced in breakdown events in this cavity. emission (and likely breakdown) sources and presented a preliminary measurement of their spectrum.

\section{ACKNOWLEDGMENTS}

We would like to thank Steve Geer and Alan Bross of Fermilab, and Bob Rimmer of JLab for help in this work. This effort was supported by the Office of High Energy Physics of the U.S. Department of Energy, under Argonne Contract No. W-31-109-ENG-38.

[1] M. Goodman et al., Lawrence Berkeley National Laboratory Report No. BNL-52623, edited by S. Ozaki, R. Palmer, M. Zisman, and J. Gallardo, 2001; http:// www.cap.bnl.gov/mumu/studyii/

[2] M. Alsharo'a et al., Phys. Rev. ST Accel. Beams 6, 081001 (2003).

[3] J. Norem, V. Wu, A. Moretti, M. Popovic, Z. Qian, L. Ducas, Y. Torun, and N. Solomey, Phys. Rev. ST Accel. Beams 6, 072001 (2003).

[4] D. Li, J. Corlett, R. MacGill, M. Zisman, J. Norem, A. Moretti, Z. Qian, J. Wallig, V. Wu, Y. Torun, and R. Rimmer, in Proceedings of the Particle Accelerator Conference, Portland, OR, 2003 (IEEE, Piscataway, New Jersey, 2003), p. 1246.

[5] J. Norem, P. Gruber, A. Bross, S. Geer, A. Moretti, Z, Qian, D. M. Kaplan, Y. Torun, D. Li, M. Zisman, and R. A. 
Rimmer, in Proceedings of the Particle Accelerator Conference, Portland, OR, 2003 (Ref. [4]), p. 1183.

[6] A. Moretti, A. D. Bross, S. Geer, Z. Qian, D. M. Errede, D. Li, J. Norem, R. A. Rimmer, Y. Torun, and M. S. Zisman, in Proceedings of LINAC 2004, Luebeck, Germany, p. 292.

[7] J. Norem, Z. Insepov, and I. Konkashbaev, Nucl. Instrum. Methods Phys. Res., Sect. A 537, 510 (2005).
[8] J. P. Barbour, W. W. Dolan, J. K. Trolan, E. E. Martin, and W. P. Dyke, Phys. Rev. 92, 45 (1953).

[9] http://www.polaroid.com

[10] P. Gruber, and Y. Torun, in Proceedings of the Particle Accelerator Conference, Portland, OR, 2003 (Ref. [4]), p. 1413.

[11] R. Rohrbach, CERN Report No. 71-28, CERN, Geneva, Switzerland, 1971. 